\title{
Mechanism of wavy vortex and sign laws in flow past a bluff body: vortex-induced vortex
}

\author{
L. M. Lin ${ }^{1} \cdot$ S. Y. Shi ${ }^{1} \cdot$ X. F. Zhong ${ }^{1} \cdot$ Y. X. $\mathrm{Wu}^{1,2}$
}

Received: 5 March 2018 / Revised: 22 April 2018 / Accepted: 3 May 2018 / Published online: 10 September 2018

(C) The Chinese Society of Theoretical and Applied Mechanics; Institute of Mechanics, Chinese Academy of Sciences and Springer-Verlag GmbH Germany, part of Springer Nature 2018

\begin{abstract}
As reported in a previous work by Lin et al. (Acta Mech Sin, 2018. https://doi.org/10.1007/s10409-018-0758-z), an interesting phenomenon was discovered based on the analysis of wavy vortex and vorticity distribution in the shear layers and near wake of a peak-perforated conic shroud, and two sign laws were summarized. In the present paper, the theory of a vortex-induced vortex is introduced to explore mechanisms in a wavy vortex and applicable sign laws for uniform and incompressible flow past a fixed bluff body. Based on the analysis of the nearest-wall flow, two vortex-induced models for streamwise and vertical vortex pairs, respectively, are proposed under two boundary cases, denoting the induced vorticity introduced or distributed on and near the walls. As a result, the first sign law, for only streamwise and vertical components of vorticity, and the second sign law, for three components of vorticity, are obtained under their own particular conditions. The first sign law reveals the intrinsic physical relationship between streamwise and vertical vorticities, independent of the distribution of spanwise vortices in the whole flow field. It is also confirmed that the spanwise vortices, as well as the shear layers and wake width, distributed wavily across the span, are attributed to the introduced streamwise or vertical vortices. The two sign laws for vorticity are independent of the disturbed spanwise wavelength and the Reynolds number. Through the analysis of flow past the conic shroud, the two sign laws are successfully used to summarize typical spacial distributions of vorticity in three flow regions: on and near the front cylinder surfaces, the separated shear layers and the near wake.
\end{abstract}

Keywords Wavy vortex $\cdot$ Sign law $\cdot$ Vortex-induced vortex $\cdot$ Wake $\cdot$ Bluff body

\section{Introduction}

Fluid flow past a bluff body is a classical problem in fluid mechanics with many engineering applications to bodies such as tall buildings, bridges and flexible risers. As a viscuous fluid encounters an obstacle, the vorticity $\omega$, defined as the curl of velocity $\boldsymbol{u}$, is first generated on solid walls, then is convected and concentrated in the upper and lower shear layers beside the bluff body. The Reynolds number is defined as $R e=U_{\infty} D / v$, where $U_{\infty}$ is the velocity of the free stream, $D$ is the character length of the bluff body and $v$ is the kinematic viscosity of the fluid. When the Reynolds number is

S. Y. Shi

shishiying@imech.ac.cn

1 Key Laboratory for Mechanics in Fluid Solid Coupling Systems, Institute of Mechanics, Chinese Academy of Sciences, Beijing 100190, China

2 School of Engineering Sciences, University of Chinese Academy of Sciences, Beijing 100049, China high enough, a vortex, usually defined as a connected fluid region with high concentration of vorticity compared with its surroundings [1], is shed alternately from both shear layers and a wake is formed. In the wake of a bluff body without any geometric disturbance, such as a circular- or square-section cylinder, the shedding vortex with the spanwise vorticity $\omega_{z}$ is two-dimensional (2-D) and laminar at $R e=40-150$ [2], known as Kármán vortex streets. Experiments and numerical simulations confirm that when a geometric disturbance is introduced on a bluff body, the spanwise vortex is wavily distorted across the span, associated to the appearance of additional components of vorticity, $\omega_{x}$ and $\omega_{y}$, in the wake $[3,4]$. This leads to the redistribution of the vorticity field and the formation of different vortex-shedding patterns, such as the $\Omega$-type vortex, and even the complete suppression of Kármán vortices. Therefore, the wavy vortex can be attributed to interactions between vortices and solid bodies, and between different vortices that play a key role in organizing the wake 

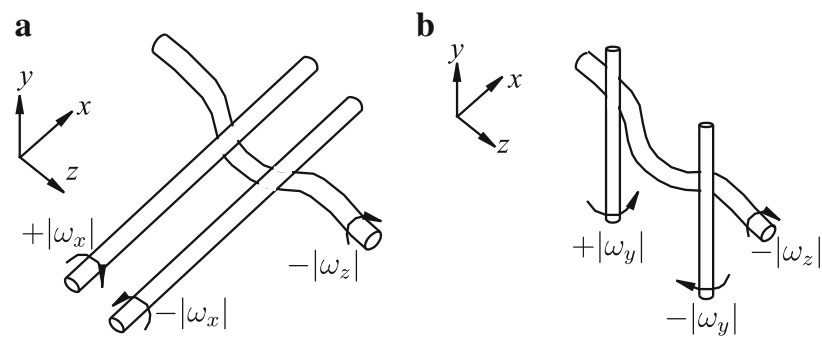

Fig. 1 Sketches of two basic interactions in the near wake of a bluff body. a Streamwise interaction between a streamwise vortex pair and a spanwise vortex. b Vertical interaction between a vertical vortex pair and a spanwise vortex

flow; this is one of the fundamental and important subjects in vorticity and vortex dynamics [5].

With regard to wavy vortices appearing in the wake of bluff bodies with geometric disturbances, two basic interactions between different vortices have recently been identified and reported [4]. As shown in Fig. 1a, the streamwise interaction between a streamwise vortex pair and a spanwise vortex, proposed by Meiburg and Lasheras [3], was investigated through experiments and numerical simulations in the plane wake behind the flat plate subjected to periodic spanwise perturbations at $R e \approx 100$. As the streamwise vortex tubes evolve, they interact with the spanwise vortices by lifting them up and pushing them down in the vertical direction (i.e. the $y$ axis) in an alternating fashion along the spanwise direction (i.e. the $z$ axis). The resulting curvature of the spanwise vortices, in turn, causes further stretching and intensification of the streamwise vorticity. Thus, the interaction between the streamwise and the spanwise vortices proceeds in a selfamplifying manner. Correspondingly, as shown in Fig. 1b, the vertical interaction between a vertical vortex pair and a spanwise vortex was studied by Lin et al. [4] through numerical calculations in the wake of the peak-perforated conic shroud at $R e=100$. Similarly, this interaction also results in the spanwise vortices twisted forward and backward in the streamwise direction (i.e. the $x$ axis) alternately along the span. Both interactions with spanwise vortices also lead to the shear layers, as well as wake width, wavily varying across the span.

However, apart from the method of introducing the above geometric disturbances, wavy spanwise vortices also appear in the wake transition of bluff bodies. When the Reynolds number exceeds 150 , two three-dimensional (3-D) instabilities successively have been found to occur, i.e. Modes A and $\mathrm{B}$, identified in experiments and direct numerical simulations [6-9]. Particularly for Mode A, the physical mechanism responsible for the wavy spanwise vortices can be attributed to the deformation of primary vortices $[10,11]$ or the appearance of vortex tongues in the vortex sheet [12].

On the other hand, through the analysis of three components of vorticity distributed in the near wake of peakperforated conic shroud at $R e=100$, an interesting physical phenomenon has been discovered [4]. As shown in Fig. 2 for the moderate effect of disturbance in regime II-B, for example in a region of $x>3$, we have $\left(+\left|\omega_{x}\right|,+\left|\omega_{y}\right|,-\left|\omega_{z}\right|\right)$ in the upper shear layer and $\left(-\left|\omega_{x}\right|,+\left|\omega_{y}\right|,+\left|\omega_{z}\right|\right)$ in the lower shear layer, as well as in the upper and lower sides of the wake, at $z=\frac{1}{4} \lambda$ (where $\lambda$ is the nondimensional wavelength of introduced conic disturbance), while $\left(-\left|\omega_{x}\right|,-\left|\omega_{y}\right|,-\left|\omega_{z}\right|\right)$ in the upper shear layer and $\left(+\left|\omega_{x}\right|,-\left|\omega_{y}\right|,+\left|\omega_{z}\right|\right)$ in the lower shear layer at $z=\frac{3}{4} \lambda$. Consequently, two sign laws can be summarized based on these four groups of vorticity sign combinations in the near wake. The first sign law asserts that across the span, $\omega_{x} \cdot \omega_{y}$ is always positive in the top shear layer but negative in the bottom shear layer. The second sign law asserts that $\omega_{x} \cdot \omega_{y} \cdot \omega_{z}$ is always negative in both shear layers. Together, these sign laws unify not only two basic interactions but also two models in explaining wavy shear layers in Refs. [13,14].

As we know now, no research and theory has sufficed to explain such a phenomenon, especially the intrinsic mechanism responsible for different vorticity components with specific signs. As for such sign laws themselves, there are still some unknowns. One question, for example, is how do these two sign laws, summarized in the shear layers and near wake, apply to the region near the front surface of a bluff body? If these sign laws near the front surface are different from those in the near wake, a second question is what conditions pertain near the front surface and the near wake? Furthermore, the more general question is whether two sign laws, obtained based on the flow past the present bluff body, also pertain to flows past other bluff bodies. At the least, we should confirmed through theoretical analysis whether this is a common case.

In present paper, the main aim is to introduce the theory of vortex-induced vortex (VIVor) as a reasonable explanation of the aforementioned two sign laws and the wavy vortex. In Sect. 2, the theory of VIVor is proposed in detail. Two kinds of basic bluff bodies are considered: flat plates and straight bluff bodies with flat stagnation surfaces. Two induced-vortex models, streamwise and vertical vortices, with two types of boundary cases, indicating the vorticity distributed on and near the walls, are analyzed. In Sect. 3, two sign laws of vorticity are theoretically verified under their own special conditions, and three sub-regions in the flow past a conic cylinder are briefly presented as an example in applying the sign laws. Finally, the conclusions are given in Sect. 4. 

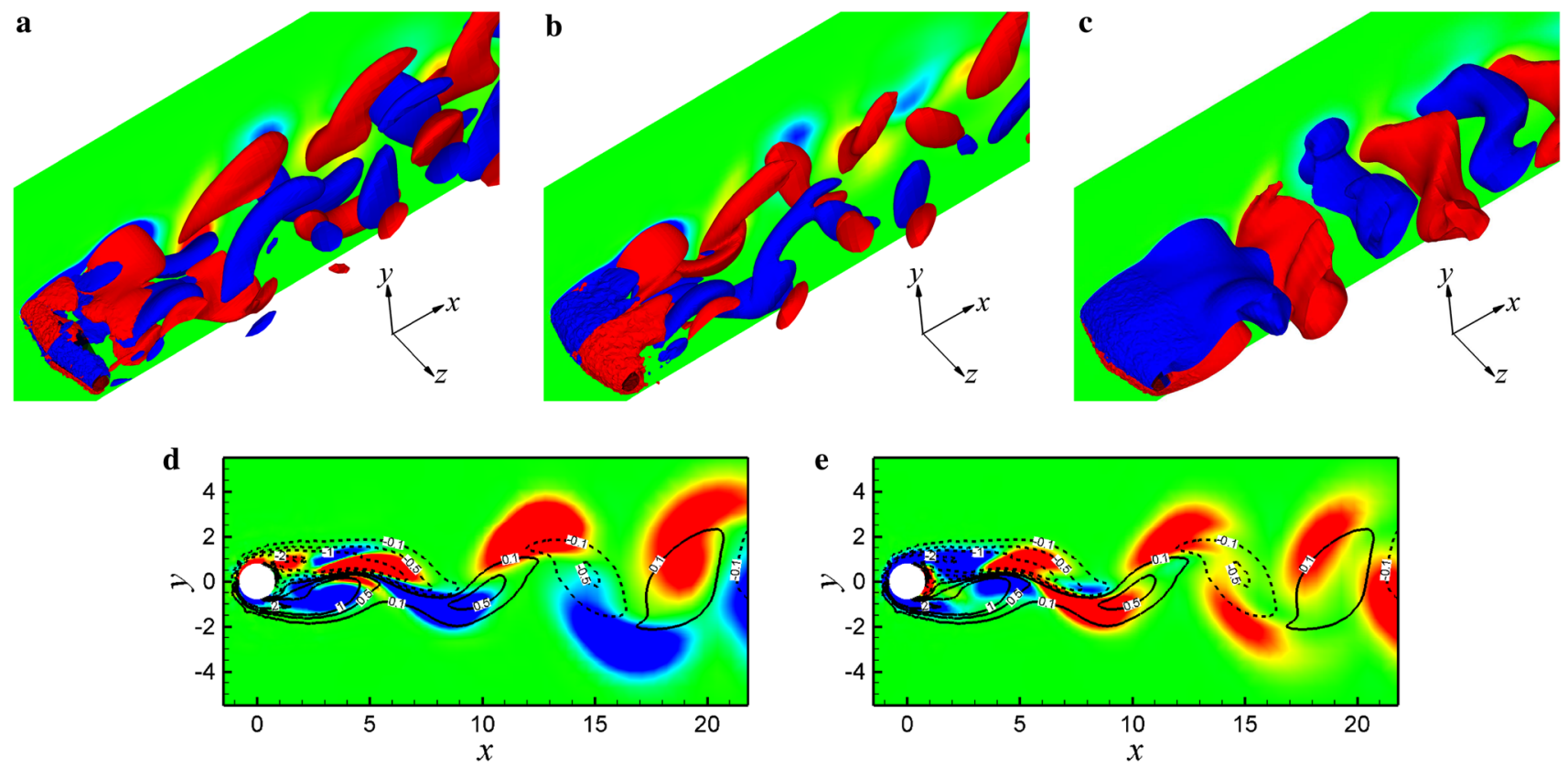

Fig. 2 In moderate disturbance regime II-B, iso-surfaces of $\mathbf{a} \omega_{x}= \pm 0.2, \mathbf{b} \omega_{y}= \pm 0.2$ and $\mathbf{c} \omega_{z}= \pm 0.2$ in the near wake and sectional contours of $\mathbf{d} \omega_{x}$ and $\mathbf{e} \omega_{y}$ at dimensionless spanwise position $z=\frac{1}{4} \lambda$ at non-dimensional time $t=600$. This diagram portrays a case of a peak-perforated conic shroud with non-dimensional wavelength $\lambda=6$, dimensionless wave height $W=0.6$, attack angle of perforation $A g=0^{\circ}$ and dimensionless diameter of holes $D o=0.4$, where red and blue colors denote positive and negative values, respectively. In the figures of iso-surfaces of vorticity, the background comprises the contours of $\omega_{z}$ at $z=0$. In plots $\mathbf{d}$ and $\mathbf{e}$, solid and dashed lines denote positive and negative values of $\omega_{z}$, respectively

\section{Theory of vortex-induced vortex (VIV or)}

According to two basic interactions in Fig. 1 and analysis of two sign laws in Fig. 2, the idea of vortex-induced vortex (VIVor) is proposed. In the VIVor theory, the vorticity field, as well as the velocity field, is induced by introducing a specific vortex (or vortex pair) under a particular boundary condition. The VIVor theory mainly provide the characteristics of vorticity signs in spacial distribution and intrinsic relationships between different components of vorticity with specific signs.

\subsection{Basic assumptions and preconditions used in the analysis}

We begin by presenting some basic assumptions and preconditions that will be important in our flow analysis.

(A1) The incoming free stream is uniform with velocity $\left(U=U_{\infty}, V=0\right)$, and incompressible with constant fluid density $\rho$ and viscosity $\mu=\rho \nu$; therefore the initial vorticity in the incoming flow is always zero.

(A2) The bluff body is fixed with zero velocity, indicating that the bluff body exerts no dynamic effect on the flow.

(A3) The body forces are conservative, e.g. the gravitational force.
(A4) The energy transportation is ignored.

(A5) The introduced vortex or vortex pair is uniformly distributed along its own rotating axis and of limited length, such as $\omega_{x}$ along the $x$ axis or $\omega_{y}$ along the $y$ axis, just as shown in Fig. 1. For the convenience of analysis, such a vortex pair is considered as having periodic distribution along the spanwise direction, as well as the induced velocity and vorticity fields.

(A6) The flow in the immediate neighborhood of the solid walls, where the viscous forces are dominant and the inertial forces can be neglected, is taken into account in our investigation of the evolution of the sign of the vorticity. The first physical reason is that the vorticity is first generated on the cylinder surfaces and then is shed with alternately shedding spanwise vortices, especially for $\omega_{x}$ due to the geometric disturbance in Fig. 2 or the wavy separation line that appears on the surface of a straight circular cylinder [15]. The second physical reason is that once such a shedding vortex and its vorticity are far away from the body, the effect of viscous forces is diffusive and dissipative, leading to the vorticity being reduced to zero under the present incoming flow. Meanwhile, in this situation, the viscous forces are negligible and the inertial forces have no effect on the evolution of the sign of the vorticity. This accords with the third reason, Kelvin's circulation theorem [16], which indicates that the circulation 


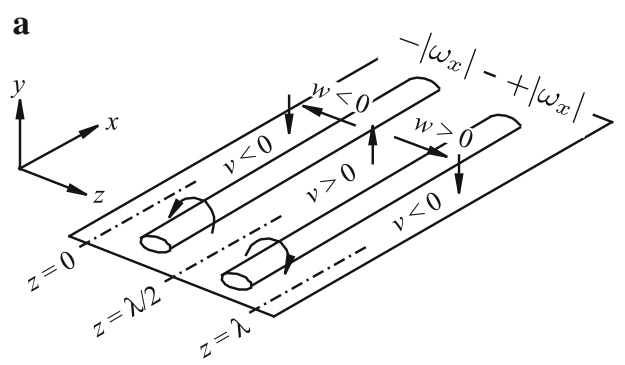

b

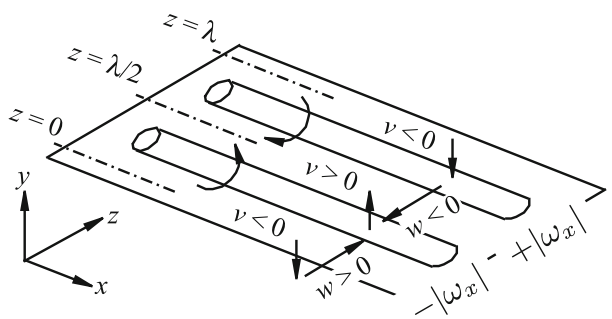

Fig. 3 Schematics of introduced streamwise vortex pair $\left(-\left|\omega_{x}\right|,+\left|\omega_{x}\right|\right)$ and induced velocity $(v, w)$ varied along the $z$ axis on or near a upper and b lower surfaces of a flat plate

and the vorticity flux for the same and single vortex with a specific sign are always invariable. Therefore, once a vortex with specially-signed vorticity is formed and shed from the bluff body, the original sign of the vorticity is almost invariant before the vortex has died away.

(A7) Consequently, the flow analysis is carried out in a local flow region, as described above, with a local Cartesian coordinate system $(x, y, z)$, in which the $x$ axis is always aligned to the local external flow, with velocity $U$ outside the region, the $z$ axis along the span of the bluff body and the $y$ axis in the normal direction of the $(x, z)$ plane.

\subsection{Governing equations}

In the above circumstances, the incompressible continuity equation in non-dimensional form is given by

$\nabla \cdot \boldsymbol{u}=0$

and the dimensionless momentum equation is written as

$\nabla p=\boldsymbol{F}+\frac{1}{R e} \nabla^{2} \boldsymbol{u}$

where the symbol $\boldsymbol{\nabla}$ is the gradient operator, $\boldsymbol{u}=(u, v, w)$ is the velocity vector, $p$ is the static pressure, $\boldsymbol{F}$ is the dimensionless conservative force vector. Velocities are scaled by the uniform free-stream velocity $U_{\infty}$ and lengths by the base height of the bluff body $D$. This formulation must be supplemented with the proper boundary conditions, expressing the absence of slip in the fluid at the still walls, i.e. the vanishing of the normal (denoted by a subscript " $n$ ") and tangential (denoted by a subscript " $\tau$ ") components of velocity:

$u_{n}=0, \quad u_{\tau}=0, \quad$ at walls.
Correspondingly, the vorticity equation is considerably simplified as

$\nabla^{2} \omega=0$

\subsection{Theoretical solutions of vortex-induced velocity and vorticity fields}

As a consequence of ignoring non-linear inertial forces, the principle of superposition of different velocity fields can be applied in solving Eq. (2). The flow field around the bluff body can be divided into two parts. One is the 2-D external flow with velocity $(U, V)$ past the bluff body without any 3-D perturbation, which mainly results in the 2-D spanwise vortices with vorticity $\Omega_{z}$ alternately being shed from the body. Another is the resultant 3-D flow field with induced velocity $(u, v, w)$ and vorticity $\left(\omega_{x}, \omega_{y}, \omega_{z}\right)$, mainly caused by the introduced vortex or vortex pair, which could be attributed to the intrinsic 3-D instability, or to the 3-D geometric disturbance or the turbulence, etc. . The theory of VIVor is focused on the induced flow field, $(u, v, w)$ and $\left(\omega_{x}, \omega_{y}, \omega_{z}\right)$, without external flow at first, and then on the coupled effects of these two parts of the flow fields, $(U+u, V+v, w)$ and $\left(\omega_{x}, \omega_{y}, \Omega_{z}+\omega_{z}\right)$.

Under assumption (A5), the two simplest induced models are subsequently established. One model is the streamwise vorticity on and near the flat plate at zero incidence, referred as the first induced model by streamwise vortices or vortex pairs, as shown in Fig. 3. Another model is the vertical vorticity on and near the flat stagnation surfaces of the straight bluff body, referred as the second induced model by vertical vortices or vortex pairs, as shown in Fig. 4.

In order to compare two different situations, two boundary cases are studied. The first boundary case is the introduced vorticity generated on the walls directly due to viscous forces, corresponding to the disturbed vortices attached on the present positions. The second boundary case is the introduced vorticity distributed near the wall and reduced to zero on the walls under the effect of viscous forces on the nearestwall region, related with the already- or early-generated 
vortices separated from the walls and transported or evolved downstream.

Because the mathematical deduction processes of velocity and vorticity equations in these two induced models coupled with two boundary cases are similar to each other, only the induced velocity and vorticity fields for the first induced model on or near the upper and lower surfaces of the flat plate, as the basic case in Fig. 3, are illustrated in detail, while the second induced model shown in Fig. 4 is just deduced individually and presented briefly.

\subsubsection{The first induced model for the flat plate}

As shown in Fig. 3 and according to assumption (A5), an introduced streamwise vortex pair with opposite signs with its center of $x=0$ is distributed uniformly along the $x$ axis on or near the upper and lower surfaces of the flat plate at zero incidence. This induced vorticity is periodic along the $z$ axis with the non-dimensional spanwise wavelength $\lambda$ and gradually disappears far away from the upper and lower surfaces, $y \rightarrow \pm \infty$, in which symbol \pm denotes signs of variables or terms for the upper and lower surfaces, successively. The induced velocities, mainly for the vertical and spanwise components, therefore independent of $x$, are expressed as

$$
\begin{gathered}
v(y, z)=-A_{v}(y) \cos \left(\frac{2 \pi}{\lambda} z\right), \\
w(y, z)=\mp A_{w}(y) \sin \left(\frac{2 \pi}{\lambda} z\right),
\end{gathered}
$$

where $A_{v}$ and $A_{w}$ are all positive and dimensionless amplitudes of $v$ and $w$, respectively, and symbol $\mp$ denotes the value signs for the upper and lower surfaces, successively. By substituting the above vertical and spanwise components of induced velocity, Eq. (5), into the continuity equation, Eq. (1), the induced streamwise velocity is obtained as

$u(x, y, z)=x\left(\frac{\mathrm{d} A_{v}}{\mathrm{~d} y} \pm \frac{2 \pi}{\lambda} A_{w}\right) \cos \left(\frac{2 \pi}{\lambda} z\right)+C_{u}$,

where $C_{u}$ is a constant, and symbol \pm denotes signs of variables for the upper and lower surfaces, successively, and the same in the following context.

Correspondingly, under the non-slip boundary conditions of Eq. (3) and the present vorticity distribution gradually disappearing away from the walls, we have:

$$
\begin{aligned}
& C_{u}=0, \\
& \left.A_{v}\right|_{y=0}=0,\left.\quad A_{v}\right|_{y \rightarrow \pm \infty}=0, \\
& \left.A_{w}\right|_{y=0}=0,\left.\quad A_{w}\right|_{y \rightarrow \pm \infty}=0, \\
& \left.\frac{\mathrm{d} A_{v}}{\mathrm{~d} y}\right|_{y=0}=0 .
\end{aligned}
$$
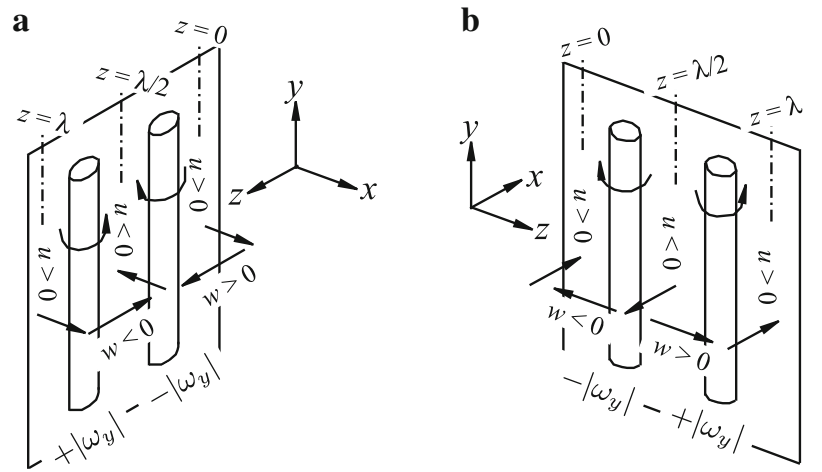

Fig. 4 Schematics of introduced vertical vortex pair $\left(-\left|\omega_{y}\right|,+\left|\omega_{y}\right|\right)$ and induced velocity $(u, w)$ varied along the $z$ axis on or near flat a rear and $\mathbf{b}$ front surfaces of the bluff body

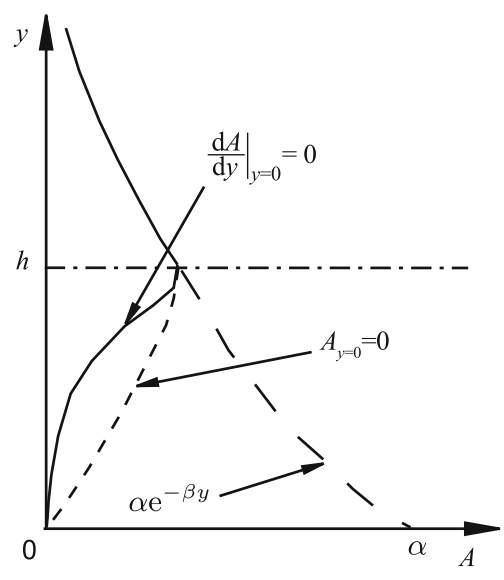

Fig. 5 Under different boundary conditions, estimation for the variation of amplitude $A$ with respect to the distance from the wall $y$, where $\alpha$ and $\beta$ are unknown constants

Before getting the exact solutions for the present model, the variation of amplitudes $A_{v}$ and $A_{w}$ with respect to the vertical position $y$ can be estimated based on the above statements, typically as shown in Fig. 5. These amplitudes first increase in the inner region, $y \in[0, \pm h]$, where the height $h$ is related to the intensity distribution of streamwise vorticity and its vertical position, and then gradually decrease in the outer region, $|y|>h$, the effect of the introduced streamwise vortex pair gradually disappearing. In the following context, the analysis and discussion are mainly carried out in this inner region, just as mentioned above in precondition (A6).

In order to avoid computing the pressure $p$, the vorticity equation, Eq. (4), is adopted with three components of induced vorticity defined as

$$
\begin{aligned}
\omega_{x} & =\frac{\partial w}{\partial y}-\frac{\partial v}{\partial z} \\
& =\left(\mp \frac{\mathrm{d} A_{w}}{\mathrm{~d} y}-\frac{2 \pi}{\lambda} A_{v}\right) \sin \left(\frac{2 \pi}{\lambda} z\right),
\end{aligned}
$$




$$
\begin{aligned}
\omega_{y} & =\frac{\partial u}{\partial z}-\frac{\partial w}{\partial x} \\
& =\frac{2 \pi}{\lambda} x\left(-\frac{\mathrm{d} A_{v}}{\mathrm{~d} y} \mp \frac{2 \pi}{\lambda} A_{w}\right) \sin \left(\frac{2 \pi}{\lambda} z\right), \\
\omega_{z} & =\frac{\partial v}{\partial x}-\frac{\partial u}{\partial y} \\
& =x\left(-\frac{\mathrm{d}^{2} A_{v}}{\mathrm{~d} y^{2}} \mp \frac{2 \pi \mathrm{d} A_{w}}{\lambda}\right) \cos \left(\frac{2 \pi}{\lambda} z\right) .
\end{aligned}
$$

Hence the vorticity equation, Eq. (4), is reduced to:

$$
\begin{aligned}
& \left(\frac{\mathrm{d}^{2}}{\mathrm{~d} y^{2}}-\frac{4 \pi^{2}}{\lambda^{2}}\right)\left(\frac{\mathrm{d} A_{w}}{\mathrm{~d} y} \pm \frac{2 \pi}{\lambda} A_{v}\right)=0, \\
& \left(\frac{\mathrm{d}^{2}}{\mathrm{~d} y^{2}}-\frac{4 \pi^{2}}{\lambda^{2}}\right)\left(\frac{\mathrm{d} A_{v}}{\mathrm{~d} y} \pm \frac{2 \pi}{\lambda} A_{w}\right)=0, \\
& \left(\frac{\mathrm{d}^{2}}{\mathrm{~d} y^{2}}-\frac{4 \pi^{2}}{\lambda^{2}}\right)\left(\frac{\mathrm{d}^{2} A_{v}}{\mathrm{~d} y^{2}} \pm \frac{2 \pi}{\lambda} \frac{\mathrm{d} A_{w}}{\mathrm{~d} y}\right)=0 .
\end{aligned}
$$

It can be seen from Eq. (9) that the $z$ component is just the spacial derivative of the $y$ component. Only $x$ and $y$ components are used to determine the unknown amplitudes $A_{v}$ and $A_{w}$ under the certain boundary case.

\section{A. The first boundary case: vorticity generated on the walls}

In the present case, the induced streamwise vorticity is distributed on the wall and reaches its maximum with the strength of $A_{\omega_{x}}(>0)$. Thus on the upper and lower surfaces, with the aid of Eqs. (7b) and (8a), we have:

$$
\left|\omega_{x}\right|_{y=0}=A_{\omega_{x}},\left.\quad \Rightarrow \quad \frac{\mathrm{d} A_{w}}{\mathrm{~d} y}\right|_{y=0}= \pm A_{\omega_{x}} .
$$

In the inner region, $y \in[0, \pm h]$, the exact solutions of Eq. (9a) and (9b) can be obtained as

$$
\begin{aligned}
& \frac{\mathrm{d} A_{w}}{\mathrm{~d} y} \pm \frac{2 \pi}{\lambda} A_{v}= \pm C_{1} \mathrm{e}^{-\frac{2 \pi}{\lambda}( \pm y)}, \\
& \frac{\mathrm{d} A_{v}}{\mathrm{~d} y} \pm \frac{2 \pi}{\lambda} A_{w}= \pm C_{2}\left[\mathrm{e}^{\frac{2 \pi}{\lambda}( \pm y)}-\mathrm{e}^{-\frac{2 \pi}{\lambda}( \pm y)}\right],
\end{aligned}
$$

where constants $C_{1}>0$ and $C_{2}>0$. Finally, the solutions for $A_{v}$ and $A_{w}$ are given by

$$
\begin{aligned}
A_{v}= & {\left[\frac{C_{2}}{2}( \pm y)-\frac{C_{1}}{\frac{8 \pi}{\lambda}}\right]\left[\mathrm{e}^{\frac{2 \pi}{\lambda}( \pm y)}-\mathrm{e}^{-\frac{2 \pi}{\lambda}( \pm y)}\right] } \\
& +\frac{C_{1}}{2}( \pm y) \mathrm{e}^{-\frac{2 \pi}{\lambda}( \pm y)} \\
A_{w}= & \frac{1}{\frac{4 \pi}{\lambda}}\left(\frac{C_{1}}{2}+C_{2}\right)\left[\mathrm{e}^{\frac{2 \pi}{\lambda}( \pm y)}-\mathrm{e}^{-\frac{2 \pi}{\lambda}( \pm y)}\right] \\
& -\frac{C_{2}}{2}( \pm y)\left[\mathrm{e}^{\frac{2 \pi}{\lambda}( \pm y)}+\mathrm{e}^{-\frac{2 \pi}{\lambda}( \pm y)}\right]
\end{aligned}
$$

$$
+\frac{C_{1}}{2}( \pm y) \mathrm{e}^{-\frac{2 \pi}{\lambda}( \pm y)}
$$

with the following conditions:

$C_{1}=A_{\omega_{x}}, \quad C_{2} \geq 2 A_{\omega_{x}}, \quad h=\frac{C_{1}}{C_{2} \frac{4 \pi}{\lambda}}$.

Accordingly, the induced streamwise velocity $u$ is obtained as

$u= \pm C_{2} x\left[\mathrm{e}^{\frac{2 \pi}{\lambda}( \pm y)}-\mathrm{e}^{-\frac{2 \pi}{\lambda}( \pm y)}\right] \cos \left(\frac{2 \pi}{\lambda} z\right)$.

Distributions of these amplitudes are represented graphically in Fig. 6a with the following specific parameters: the constant $C_{2}=2 A_{\omega_{x}}$, the different wavelengths $\lambda=\pi$, $2 \pi$ and $4 \pi$, and therefore the resulting different heights $h_{\lambda=\pi}=0.125, h_{\lambda=2 \pi}=0.25$ and $h_{\lambda=4 \pi}=0.5$. As the normal distance $y$ away from the walls increases, $A_{w}$ is almost linearly increased, while $A_{v}$ is gradually increased non-linearly. In the case of vorticity generated on the walls with maximal intensity, $A_{w}$ is obviously greater than $A_{v}$ at the same height and wavelength, and as the wavelength of the disturbance increases, $A_{v}$ is reduced quickly at the same height, while $A_{w}$ is increased only a little.

Meanwhile, the induced pressure $p$ for the upper surface is presented as

$$
\begin{aligned}
p(y, z)= & -\frac{C_{2}}{R e}\left(\mathrm{e}^{\frac{2 \pi}{\lambda} y}-\mathrm{e}^{-\frac{2 \pi}{\lambda} y}\right) \cos \left(\frac{2 \pi}{\lambda} z\right) \\
& -\frac{C_{1}}{R e} \mathrm{e}^{-\frac{2 \pi}{\lambda} y} \cos \left(\frac{2 \pi}{\lambda} z\right)+C_{p},
\end{aligned}
$$

where $C_{p}$ is a constant.

The distribution of induced vorticity is thus obtained from Eq. (8) as follows,

$$
\begin{aligned}
& \omega_{x}=-C_{1} \mathrm{e}^{-\frac{2 \pi}{\lambda}( \pm y)} \sin \left(\frac{2 \pi}{\lambda} z\right), \\
& \omega_{y}=\mp C_{2} \frac{2 \pi}{\lambda} x\left[\mathrm{e}^{\frac{2 \pi}{\lambda}( \pm y)}-\mathrm{e}^{-\frac{2 \pi}{\lambda}( \pm y)}\right] \sin \left(\frac{2 \pi}{\lambda} z\right), \\
& \omega_{z}=-C_{2} \frac{2 \pi}{\lambda} x\left[\mathrm{e}^{\frac{2 \pi}{\lambda}( \pm y)}+\mathrm{e}^{-\frac{2 \pi}{\lambda}( \pm y)}\right] \cos \left(\frac{2 \pi}{\lambda} z\right) .
\end{aligned}
$$

\section{B. The second boundary case: vorticity distributed near the walls}

In the present case, the vorticity is already generated upstream, convected into and distributed just above the present positions, and finally disappears on the surfaces. Such boundary conditions can be expressed on the basis of Eqs. (7b) and (8a) by

$\left|\omega_{x}\right|_{y=0}=0,\left.\quad \Rightarrow \quad \frac{\mathrm{d} A_{w}}{\mathrm{~d} y}\right|_{y=0}=0$. 
a

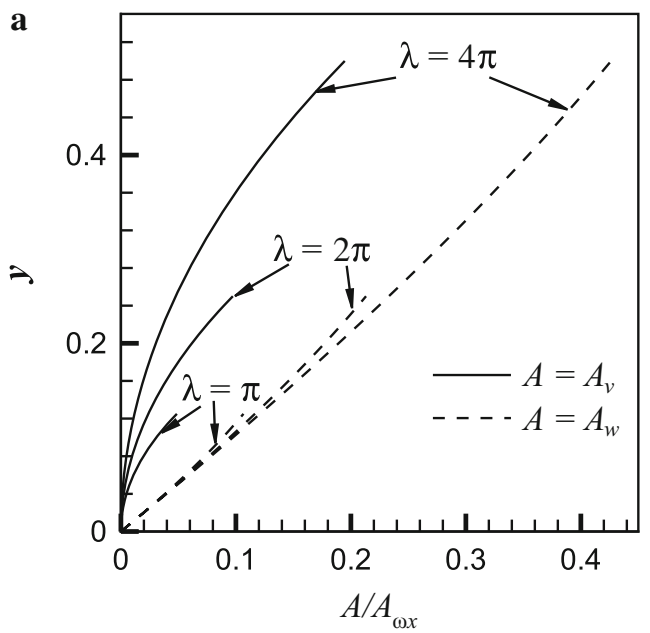

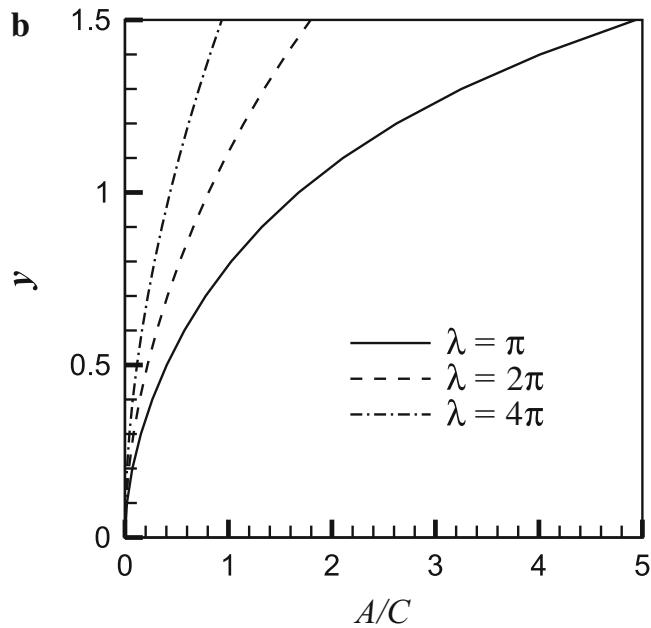

Fig. 6 Within the inner region, variations of relative amplitudes. a $A_{v}$ and $A_{w}$ divided by $A_{\omega_{x}}$ in the first boundary case with $C_{2}=2 A_{\omega_{x}}$. b $A$ divided by $C$ in the second boundary case, with respect to the distance away from the surface, $y$, and different wavelength of disturbance $\lambda$ in the first induced model

By comparison with the boundary condition of $A_{v}$, Eq. (7d), we can draw a conclusion from the above system of vorticity equations, Eq. (9), that variations of $A_{v}$ and $A_{w}$ are exactly identical. The solutions in the inner region, $y \in[0, \pm h]$, are given by

$A_{v}=A_{w}=A$,

$\frac{\mathrm{d} A}{\mathrm{~d} y} \pm \frac{2 \pi}{\lambda} A=C\left(\mathrm{e}^{\frac{2 \pi}{\lambda} y}-\mathrm{e}^{-\frac{2 \pi}{\lambda} y}\right)$,

where $C$ is also a positive constant. The distribution of amplitude is obtained as

$A=\frac{C}{\frac{4 \pi}{\lambda}}\left[\mathrm{e}^{\frac{2 \pi}{\lambda}( \pm y)}-\mathrm{e}^{-\frac{2 \pi}{\lambda}( \pm y)}\right]-C( \pm y) \mathrm{e}^{-\frac{2 \pi}{\lambda}( \pm y)}$,

and illustrated in Fig. 6b with different wavelengths, showing that its variation, along with the vertical height and wavelength, is similar to that in the first boundary case, just as shown in Fig. 6a.

As a result, the induced streamwise velocity $u$ is obtained as

$u= \pm C x\left[\mathrm{e}^{\frac{2 \pi}{\lambda}( \pm y)}-\mathrm{e}^{-\frac{2 \pi}{\lambda}( \pm y)}\right] \cos \left(\frac{2 \pi}{\lambda} z\right)$

exactly similar to that in the first boundary case, Eq. (14).

The induced pressure $p$ for the upper surface is also given by

$p(y, z)=\frac{2 C}{R e} \mathrm{e}^{-\frac{2 \pi}{\lambda} y} \cos \left(\frac{2 \pi}{\lambda} z\right)+C_{p}$,

where $C_{p}$ is a constant.
In the present boundary case, the induced vorticity field is then presented as

$$
\begin{aligned}
& \omega_{x}=-C\left[\mathrm{e}^{\frac{2 \pi}{\lambda}( \pm y)}-\mathrm{e}^{-\frac{2 \pi}{\lambda}( \pm y)}\right] \sin \left(\frac{2 \pi}{\lambda} z\right), \\
& \omega_{y}=\mp C \frac{2 \pi}{\lambda} x\left[\mathrm{e}^{\frac{2 \pi}{\lambda}( \pm y)}-\mathrm{e}^{-\frac{2 \pi}{\lambda}( \pm y)}\right] \sin \left(\frac{2 \pi}{\lambda} z\right), \\
& \omega_{z}=-C \frac{2 \pi}{\lambda} x\left[\mathrm{e}^{\frac{2 \pi}{\lambda}( \pm y)}+\mathrm{e}^{-\frac{2 \pi}{\lambda}( \pm y)}\right] \cos \left(\frac{2 \pi}{\lambda} z\right) .
\end{aligned}
$$

\subsubsection{The second induced model for the straight bluff body}

In a similar way, the induced vorticity distribution is assumed in Fig. 4, and thus the induced velocity field with the help of non-slip boundary conditions on stagnation surfaces and the continuity equation is assumed as follows:

$u(x, z)=A_{u}(x) \cos \left(\frac{2 \pi}{\lambda} z\right)$,

$v(x, y, z)=-y\left(\frac{\mathrm{d} A_{u}}{\mathrm{~d} x} \pm \frac{2 \pi}{\lambda} A_{w}\right) \cos \left(\frac{2 \pi}{\lambda} z\right)$,

$w(x, z)= \pm A_{w}(x) \sin \left(\frac{2 \pi}{\lambda} z\right)$,

where symbol \pm denotes signs of variables or terms for the rear and front stagnation surfaces, successively. Similar boundary conditions of amplitudes are

$\left.A_{u}\right|_{x=0}=0,\left.\quad \frac{\mathrm{d} A_{u}}{\mathrm{~d} x}\right|_{x=0}=0,\left.\quad A_{w}\right|_{x=0}=0$,

where $A_{u}$ and $A_{w}$ are dimensionless amplitudes of $u$ and $w$, respectively. It should be noted that the origin of the coordinate, i.e. $y=0$, is set on the center of the surface in the $y$ 
direction. As for the finite height of the bluff body, $y=0$ is located at the center of body.

\section{A. The first boundary case: vorticity generated on the walls}

By solving Eq. (4) in a similar manner, the solutions in the present case in which the induced vertical vorticity is generated on the wall with the amplitude of $A_{\omega_{y}}$ are obtained as

$$
\begin{aligned}
A_{u}= & {\left[\frac{C_{2}}{2}( \pm x)-\frac{C_{1}}{\frac{8 \pi}{\lambda}}\right]\left[\mathrm{e}^{\frac{2 \pi}{\lambda}( \pm x)}-\mathrm{e}^{-\frac{2 \pi}{\lambda}( \pm x)}\right] } \\
& +\frac{C_{1}}{2}( \pm x) \mathrm{e}^{-\frac{2 \pi}{\lambda}( \pm x)} \\
A_{w}= & \frac{1}{\frac{4 \pi}{\lambda}}\left(\frac{C_{1}}{2}+C_{2}\right)\left[\mathrm{e}^{\frac{2 \pi}{\lambda}( \pm x)}-\mathrm{e}^{-\frac{2 \pi}{\lambda}( \pm x)}\right] \\
& -\frac{C_{2}}{2}( \pm x)\left[\mathrm{e}^{\frac{2 \pi}{\lambda}( \pm x)}+\mathrm{e}^{-\frac{2 \pi}{\lambda}( \pm x)}\right] \\
& +\frac{C_{1}}{2}( \pm x) \mathrm{e}^{-\frac{2 \pi}{\lambda}( \pm x)}
\end{aligned}
$$

where symbol \pm denotes signs of variables for the rear and front stagnation surfaces, successively, and in the inner region, $x \in[0, \pm h]$, with conditions

$C_{1}=A_{\omega_{y}}, \quad C_{2} \geq 2 A_{\omega_{y}}, \quad h=\frac{C_{1}}{C_{2} \frac{4 \pi}{\lambda}}$.

This could be also deduced through the coordinate transformation by replacing $y$ with $x$ in Eq. (12). Variations of amplitudes are also identical to those in Fig. 6a with respect to $x$, rather than $y$. The induced vertical velocity $v$ is obtained as

$v=\mp C_{2} y\left[\mathrm{e}^{\frac{2 \pi}{\lambda}( \pm x)}-\mathrm{e}^{-\frac{2 \pi}{\lambda}( \pm x)}\right] \cos \left(\frac{2 \pi}{\lambda} z\right)$,

where both $\mp$ and \pm symbols denote signs for the rear and front stagnation surfaces successively, the same as in the following context.

The induced vorticity is then obtained as

$$
\begin{aligned}
& \omega_{x}=\mp C_{2} \frac{2 \pi}{\lambda} y\left[\mathrm{e}^{\frac{2 \pi}{\lambda}( \pm x)}-\mathrm{e}^{-\frac{2 \pi}{\lambda}( \pm x)}\right] \sin \left(\frac{2 \pi}{\lambda} z\right), \\
& \omega_{y}=-C_{1} \mathrm{e}^{-\frac{2 \pi}{\lambda}( \pm x)} \sin \left(\frac{2 \pi}{\lambda} z\right), \\
& \omega_{z}=-C_{2} \frac{2 \pi}{\lambda} y\left[\mathrm{e}^{\frac{2 \pi}{\lambda}( \pm x)}+\mathrm{e}^{-\frac{2 \pi}{\lambda}( \pm x)}\right] \cos \left(\frac{2 \pi}{\lambda} z\right) .
\end{aligned}
$$

\section{B. The second boundary case: vorticity distributed near the walls}

In the present boundary case, the amplitudes $A_{u}$ and $A_{w}$ are given by

$$
\begin{aligned}
A_{u}=A_{w}= & \frac{C}{\frac{4 \pi}{\lambda}}\left[\mathrm{e}^{\frac{2 \pi}{\lambda}( \pm x)}-\mathrm{e}^{-\frac{2 \pi}{\lambda}( \pm x)}\right] \\
& -C( \pm x) \mathrm{e}^{-\frac{2 \pi}{\lambda}( \pm x)} .
\end{aligned}
$$

The induced vertical velocity $v$ is presented as

$v=\mp C y\left[\mathrm{e}^{\frac{2 \pi}{\lambda}( \pm x)}-\mathrm{e}^{-\frac{2 \pi}{\lambda}( \pm x)}\right] \cos \left(\frac{2 \pi}{\lambda} z\right)$.

Correspondingly, the induced vorticity is presented as follows:

$\omega_{x}=\mp C \frac{2 \pi}{\lambda} y\left[\mathrm{e}^{\frac{2 \pi}{\lambda}( \pm x)}-\mathrm{e}^{-\frac{2 \pi}{\lambda}( \pm x)}\right] \sin \left(\frac{2 \pi}{\lambda} z\right)$,
$\omega_{y}=-C\left[\mathrm{e}^{\frac{2 \pi}{\lambda}( \pm x)}-\mathrm{e}^{-\frac{2 \pi}{\lambda}( \pm x)}\right] \sin \left(\frac{2 \pi}{\lambda} z\right)$,
$\omega_{z}=-C \frac{2 \pi}{\lambda} y\left[\mathrm{e}^{\frac{2 \pi}{\lambda}( \pm x)}+\mathrm{e}^{-\frac{2 \pi}{\lambda}( \pm x)}\right] \cos \left(\frac{2 \pi}{\lambda} z\right)$.

\section{Effect of finite vertical length of the bluff body}

Now let us discuss the effect of the finite vertical length of the bluff body $D$ on the above analysis. Here, the local external flow with velocity $(U, V)$ is still excluded. The local flow region near the rear surface is taken as an example. It can be seen from Eqs. (27) and (30) that near the rear stagnation surface denoted by the + sign the induced vertical velocity can be expressed approximately by

$v \propto-y\left(\mathrm{e}^{\frac{2 \pi}{\lambda} x}-\mathrm{e}^{-\frac{2 \pi}{\lambda} x}\right) \cos \left(\frac{2 \pi}{\lambda} z\right)$,

in the inner region $x \in[0, h]$, regardless of vertical vorticity distributed on or near the wall. The vertical length scale of (almost) uniformly distributed vertical vorticity is almost equivalent to the finite length scale of body $D$. This means that the induced velocity $(u, v, w)$ would be gradually reduced down to zero far away from the rear surface or shear layers, as shown in Fig. 7, due to the gradual disappearance of the effect of the introduced vertical vortex pair. The typical distributions of $v$ and $w$ can be shown in Fig. 7 at different spanwise positions. Therefore, it shows that $\omega_{x}$ still has a negative sign because of $\partial w / \partial y<0$ and $\partial v / \partial z>0$, for instance, in local region $(y>0,0<z<\lambda / 2)$, and its qualitative distribution is also demonstrated in Fig. 7. It can be concluded that the finite vertical height of the bluff body has little effect on the qualitative distributions of induced vertical velocity and streamwise vorticity. The maximal value of $\omega_{x}$ would appear in the shear layers where $|v|$ reaches the local maximum, as shown in Fig. 7. 

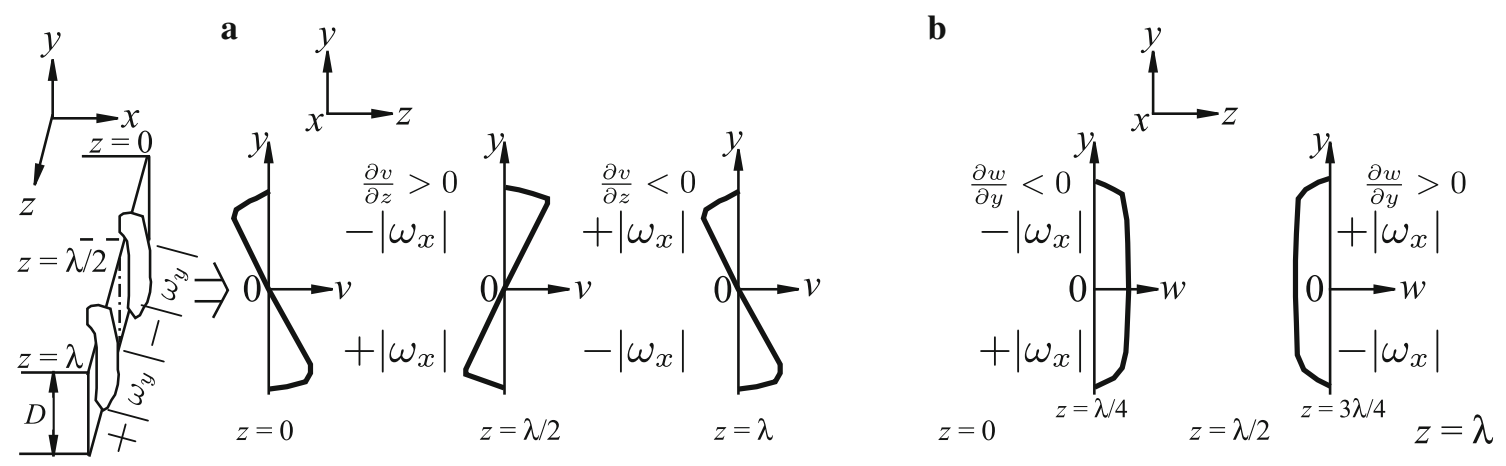

Fig.7 Qualitative distributions of induced velocities, $\mathbf{a} v$ and $\mathbf{b} w$, and streamwise vorticity, $\omega_{x}$, near the rear surface of bluff body with the finite height $D$ in the second induced model

\section{Results}

In the following analysis, the Cartesian coordinate system $(X, Y, Z)$, where the $X$ axis is aligned with the incoming free stream, the $Z$ axis is the span of the bluff body and the $Y$ axis is perpendicular to the $(X, Z)$ plane, in flow past the bluff body, is consistent with the local Cartesian coordinate system $(x, y, z)$ used in two induced models, because the local external flow is taken as the incoming free stream. Therefore, in the flow past the bluff body with or without geometric disturbances, the position of $x=0$ denotes either the center of introduced streamwise vortices in the first induced model or the center of the bluff body along the streamwise direction. While the position of $y=0$ denotes either the center of introduced vertical vortices in the second induced model or the center of the bluff body or wake center plane (WCP) along the vertical direction. Then the region $x<0$ denotes the flow on and near the front surface of the bluff body, while the region $x>0$ is the flow away from the rear surface. The upper shear layer or upside of WCP is denoted by $y>0$, and the lower shear layer or downside of WCP by $y<0$.

Under present circumstances, only three sub-regions, i.e. on and near front cylinder surfaces (R-I), separated shear layers (R-II) and near wake (R-III), are presented to illustrate the application of the sign laws for vorticity components. Because the local external flow is opposite to the incoming free stream, the local reversed flow regions on both lateral sides and the recirculation behind the body are not taken into account in this paper, but may be discussed in future work.

\subsection{The first sign law for streamwise and vertical components of vorticity}

Based on the above analysis of spacial distributions of streamwise and vertical components of vorticity under different circumstances, such as the first boundary case (f.b.c.) and the second boundary case (s.b.c.), the first sign law is obtained. For example, in the first induced model of stream- wise vortex pairs, we have

$$
\begin{aligned}
& \omega_{x} \cdot \omega_{y} \\
& =C_{1} C_{2} \frac{2 \pi}{\lambda} x \mathrm{e}^{-\frac{2 \pi}{\lambda} y}\left(\mathrm{e}^{\frac{2 \pi}{\lambda} y}-\mathrm{e}^{-\frac{2 \pi}{\lambda} y}\right) \sin ^{2}\left(\frac{2 \pi}{\lambda} z\right),
\end{aligned}
$$

in f.b.c.

$\omega_{x} \cdot \omega_{y}=C^{2} \frac{2 \pi}{\lambda} x\left(\mathrm{e}^{\frac{2 \pi}{\lambda} y}-\mathrm{e}^{-\frac{2 \pi}{\lambda} y}\right)^{2} \sin ^{2}\left(\frac{2 \pi}{\lambda} z\right)$,

in s.b.c.

on the upper surface of the flat plate in the inner region, $y \in[0, h]$, and

$\omega_{x} \cdot \omega_{y}=-C_{1} C_{2} \frac{2 \pi}{\lambda} x \mathrm{e}^{\frac{2 \pi}{\lambda} y}\left(\mathrm{e}^{-\frac{2 \pi}{\lambda} y}-\mathrm{e}^{\frac{2 \pi}{\lambda} y}\right) \sin ^{2}\left(\frac{2 \pi}{\lambda} z\right)$,

in f.b.c.

$\omega_{x} \cdot \omega_{y}=-C^{2} \frac{2 \pi}{\lambda} x\left(\mathrm{e}^{-\frac{2 \pi}{\lambda} y}-\mathrm{e}^{\frac{2 \pi}{\lambda} y}\right)^{2} \sin ^{2}\left(\frac{2 \pi}{\lambda} z\right)$,

in s.b.c.

on the lower surface within the region $y \in[-h, 0]$. We can rewrite these as follows,

$\omega_{x} \cdot \omega_{y} \geq 0$

if $(x \geq 0, y \geq 0)$ or $(x \leq 0, y \leq 0)$, or $(x y \geq 0)$, and

$\omega_{x} \cdot \omega_{y} \leq 0$

if ( $x \geq 0, y \leq 0)$ or $(x \leq 0, y \geq 0)$, or $(x y \leq 0)$. This relationship can also be obtained for the second induced model, Eqs. (28) and (31). It shows that the first sign law is independent of the distribution of induced spanwise vorticity $\omega_{z}$ and the two induced models. Moreover, it is also unrelated to the non-dimensional wavelength $\lambda$ and Reynolds number $R e$. Thus it can be concluded that the first sign law is an intrinsic physical mechanism of fluid mechanics under any kind of disturbance. 


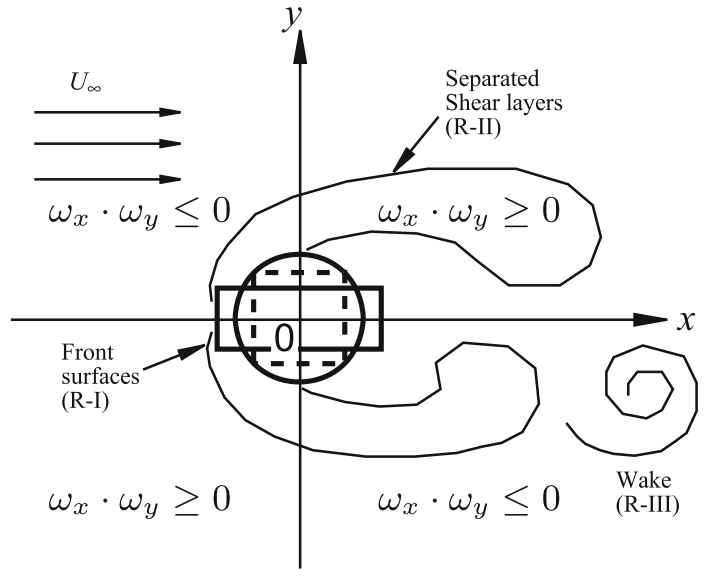

Fig. 8 Schematics of the first sign law for streamwise and vertical components of vorticity $\left(\omega_{x} \cdot \omega_{y}\right)$ distributed in the flow past the bluff body, typically such as the circular (solid line), or square (dashed line), or rectangular (solid line) cylinder placed in the center of the coordinate system. Typically, three flow regions are of main concern, i.e. front cylinder surfaces (R-I), separated shear layers (R-II) and near wake (R-III), respectively

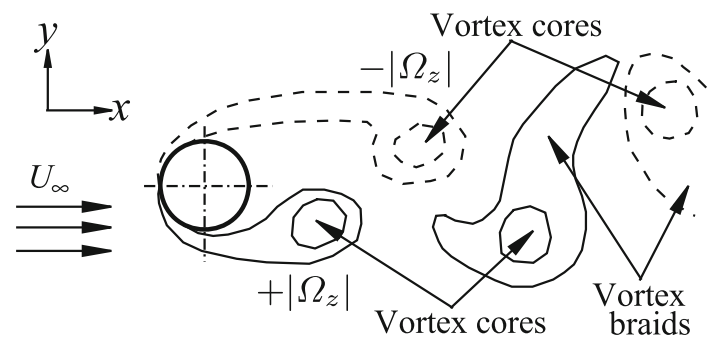

Fig. 9 Schematics of typical distribution of spanwise vorticity $\Omega_{z}$ in the 2-D wake flow past a circular-section cylinder, where solid and dashed lines denote positive and negative values, respectively

As indicated in the above analysis (A6), we can apply the first sign law in three sub-regions, as sketched in Fig. 8 for fixed bluff bodies typically with circular, square and rectangular cross-sections. It is theoretically verified that the sign of $\omega_{x} \cdot \omega_{y}$ is positive in the upper shear layer but negative in the lower shear layer. Interestingly, this feature is just opposite on and near the front surface. It should be noted that the sub-region of $x<0$ in the first sign law would reach downstream into the shear layers at a certain distance due to the effect of inertial forces from the incoming free stream.

In the flow past the bluff body, we can summarize the first sign law by introducing the sign function, $\operatorname{sgn}(x)$, and the first sign variable, $\varpi_{1}=\omega_{x} \cdot \omega_{y}$, except some specific positions (e.g. $z=0, \frac{1}{2} \lambda$ and $\lambda$ ), at which $\varpi_{1}=0$, as

$\operatorname{sgn}\left(\omega_{x} \cdot \omega_{y}\right)=\operatorname{sgn}\left(\varpi_{1}\right)=\left\{\begin{aligned}-1, & x y<0, \\ 1, & x y>0 .\end{aligned}\right.$

\subsection{The second sign law for three components of vorticity}

In the 2-D wake flow of the fixed bluff body without any disturbance at lower Reynolds numbers, the typical distribution of spanwise vorticity $\Omega_{z}$ near the front surface, shear layers and near wake is first summarized as shown in Fig. 9. Generally, the sign of $\Omega_{z}$ is negative at $y>0$ and positive $y<0$, except in the vortex braids, which are stretched into the opposite side of WCP by the downstream spanwise vortex with the opposite sign. From the viewpoint of the time-averaged profile of streamwise velocity or wake profile, this feature can be simply expressed by

$\operatorname{sgn}\left(\Omega_{z}\right)=\left\{\begin{array}{rr}1, & y<0 \\ -1, & y>0\end{array}\right.$

The induced spanwise vorticity $\omega_{z}$ due to the introduction of streamwise or vertical vorticity is assumed to be smaller in magnitude than the above spanwise vorticity $\Omega_{z}$, i.e. $\left|\omega_{z}\right|<\left|\Omega_{z}\right|$. Consequently, the appearance of $\omega_{z}$ is just the modification of $\Omega_{z}$ and leads to the latter wavily varying along the span, as well as the shear layers and wake width. Therefore, we have the following sign feature of coupled spanwise vorticity:

$\operatorname{sgn}\left(\Omega_{z}+\omega_{z}\right)=\left\{\begin{array}{rr}1, & y<0 \\ -1, & y>0 .\end{array}\right.$

Based on this analysis and in consideration of the first sign law, Eqs. (39) and (41), the second sign laws can be obtained for the flow past the bluff body with three components of vorticity by introducing the second sign variable, $\varpi_{2}=\omega_{x}$. $\omega_{y} \cdot\left(\Omega_{z}+\omega_{z}\right)$, except some special positions (e.g. $z=0$, $\frac{1}{2} \lambda$ and $\lambda$ ) at which $\varpi_{2}=0$,

$\operatorname{sgn}\left(\varpi_{2}\right)=\left\{\begin{aligned} 1, & x<0, \\ -1, & x>0 .\end{aligned}\right.$

This theoretical relationship of the coupled vorticity field shows that the sign of combination of all three components of vorticity is always positive near the front surface $(x<0)$ but negative in the shear layers and near wake $(x>0)$. As a matter of fact, as denoted in the first sign law, the sub-region of $x<0$ in the second sign law would reach downstream into the shear layers at a certain distance due to the effect of inertial forces from the incoming free stream. Furthermore, the second sign law is also independent of control parameters $\lambda$ and $R e$, and of two induced models in the present circumstances.

The wake flow, various vortex-shedding patterns and their physical mechanisms are mainly concerned, as shown in Fig. 9, because the flow in region $x<0$ is usually simpler 
than that in the near wake. Here, the second sign law in the wake is referred to as the negative (second) sign law for the vorticity field in the wake of the fixed bluff body. Here $\omega_{x}$ and $\omega_{y}$ can be explained as a result of three-dimensional geometric disturbance or instability, and then are transported into the shear layers and near wake. These additional components of vorticity, $\omega_{x}$ and $\omega_{y}$, therefore play an important role in the main vortex-shedding pattern, like the hairpin-like vortex in the wake of a wavy square-section cylinder [13] or the Пtype vortex in the wake of a straight square-section cylinder [4], in which the head line, "_-", denotes the spanwise vortex, while two legs, "| |", denote streamwise and/or vertical vortex pairs shed with this spanwise vortex and elongated by the upstream spanwise vortex of opposite sign.

From Eq. (42) and the definition of the second sign variable, we can obtain the relationship between the first and second sign laws. Because of $\varpi_{2}=\varpi_{1} \cdot\left(\Omega_{z}+\omega_{z}\right)$, we have the following relationship

$\operatorname{sgn}\left(\varpi_{1} \cdot \varpi_{2}\right)=\operatorname{sgn}\left(\Omega_{z}+\omega_{z}\right)=\left\{\begin{aligned} 1, & y<0 \\ -1, & y>0\end{aligned}\right.$

This indicates that the sign of the first sign variable is just opposite to the sign of the second sign variable above WCP $y>0$, but the same as the sign of the second sign variable under WCP $y<0$. This relationship is attributed to the sign of total spanwise vorticity typically distributed on and near the bluff body.

\subsection{The third sign law for streamwise and vertical components of velocity}

Interestingly, similar to the first sign law for $\omega_{x}$ and $\omega_{y}$, the sign law for streamwise and vertical components of induced velocity, $u$ and $v$, can also be found. Based on Eqs. (5a) and (14) or (20) for the upper and lower surfaces due to the introduced streamwise vortex pair, and Eqs. (23a) and (27) or (30) for both stagnation surfaces due to the introduced vertical vortex pair, the third sign law is summarized in the following form by introducing the third sign variable, $\varpi_{3}=$ $u \cdot v$, also independent of $\lambda$ and $R e$,

$\operatorname{sgn}(u \cdot v)=\operatorname{sgn}\left(\varpi_{3}\right)=\left\{\begin{aligned} 1, & x y<0, \\ -1, & x y>0,\end{aligned}\right.$

with the exception of some special positions, e.g. $z=\frac{1}{4} \lambda$ and $\frac{3}{4} \lambda$, at which $\varpi_{3}=0$.

Furthermore, Eq. (44) shows that the third sign law is independent of the distribution of induced spanwise velocity $w$ and influence of external flow, also indicating that this relationship is a physical mechanism intrinsic to the flow past the bluff body. Because $\omega_{x}$ and $\omega_{y}$ originate from the 3 -D disturbed flow field and do not exist in the 2-D external flow field (except the turbulence), the first sign law is easily demonstrated by iso-surfaces of vorticity. However, the third sign law is difficult to illustrate by iso-surfaces of velocity due to the difficulty in distinguishing the induced velocities $u$ and $v$ from the 2-D external flow velocity. However, from the viewpoint of turbulent flow, in which the induced velocity is assumed to be the velocity of fluctuation due to the turbulence, some physical phenomena maybe be better understood or explained; these may be studied and reported in future work.

\subsection{An example: flow past the conic cylinder at $\operatorname{Re}=100$}

In this sub-section, a typical example, the flow past a conic cylinder at $R e=100$ [4], is selected to illustrate the characteristics of the two sign laws for vorticity components in three sub-regions, i.e. R-I, R-II and R-III. It has already been reported [4] that once the geometric disturbances are introduced, alternately shedding spanwise vortices are wavily distorted across the span by effects of streamwise and vertical vortices, similar to Mode $\mathrm{A}$ in the 3 -D wake transition of the square-section cylinder, as shown in Fig. 10, even totally suppressed at certain control parameters, as shown in Fig. 11. Here, these two cases are taken into account in the analysis of the vorticity sign. It should be noted that, for the convenience of simplification in symbols of vorticity, three components of vorticity, $\omega_{x}, \omega_{y}$ and $\omega_{z}$, in the following context, generally do not denote three induced vorticity components again, but denote three total vorticity components, including induced vorticity components, particularly for spanwise vorticity.

First, let us check the vorticity distributions in region RI. For example in both cases, Figs. 10 and 11, the sign of $\omega_{x}$ is positive in the region of $\left(y>0,0<z<\frac{1}{2} \lambda\right)$ but negative in the region of ( $y>0, \frac{1}{2} \lambda<z<\lambda$ ), while the sign of $\omega_{y}$ is negative and positive, respectively. This is in perfect agreement with the first sign law in region $y>0$, and similarly in region $y<0$. Under the specific distribution of spanwise vorticity, as shown in Figs. 10c or 11c, the second sign variable is always positive, consistent with the second sign law in region $x<0$. As shown in Figs. 10d, e and $11 \mathrm{~d}$, e, the region of vorticity combinations in the first and second sign laws extends into the upper and lower shear layers downstream about $3 D$ and $2 D$, respectively, attributed to the convective effect of inertial forces from the incoming free stream, as stated before.

Then in region R-II, except the above region influenced by sign laws at $x<0$, we can summarize the specific features of vorticity components as follows. In the upper shear layer, the sign of $\omega_{x}$ is mainly positive and negative across the span, as is the sign of $\omega_{y}$. However, in the lower shear layer, the sign of 

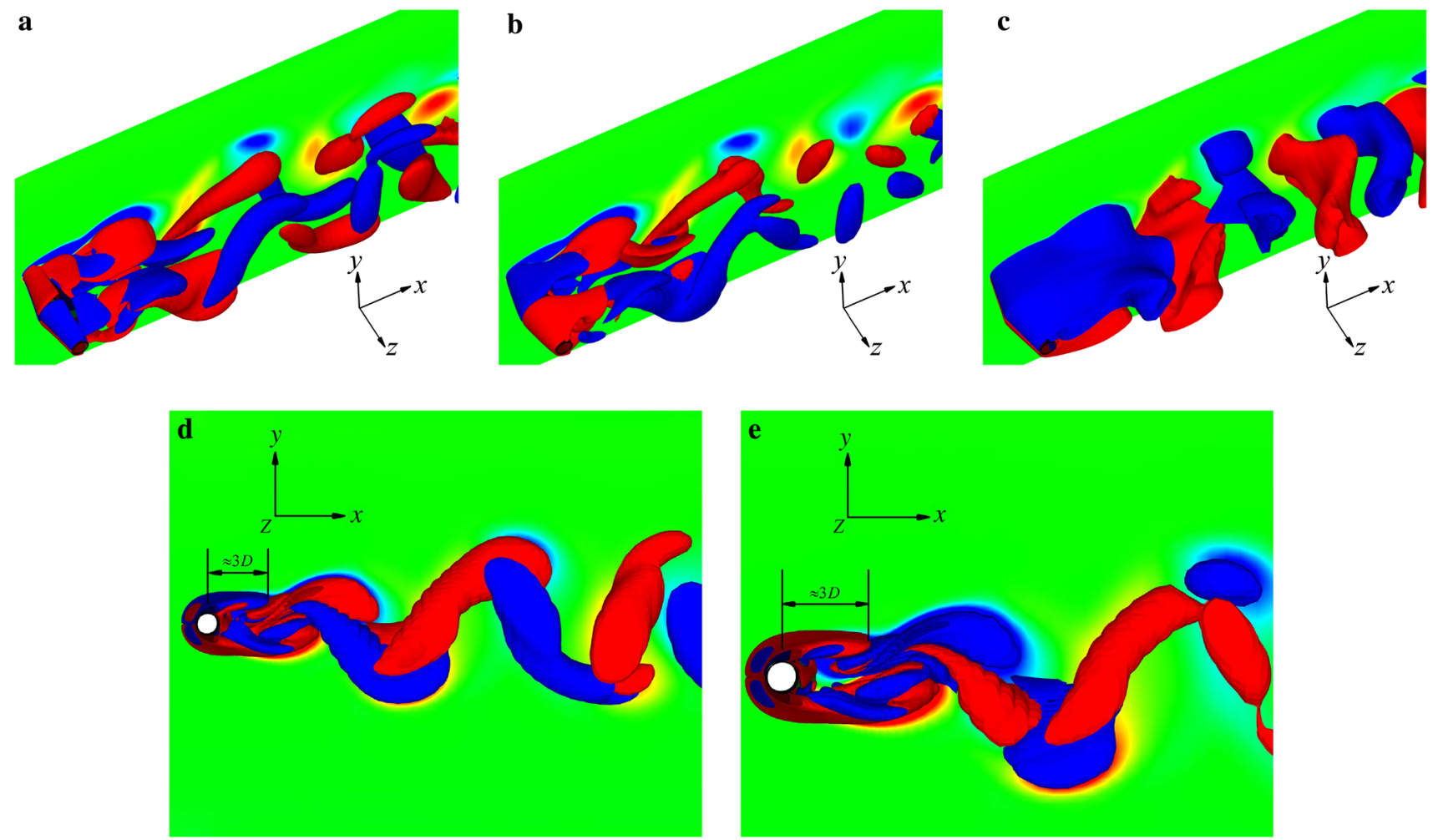

Fig. 10 Iso-surfaces of $\mathbf{a} \omega_{x}= \pm 0.2, \mathbf{b} \omega_{y}= \pm 0.2, \mathbf{c} \omega_{z}= \pm 0.2, \mathbf{d} \varpi_{1}= \pm 0.01$ and $\mathbf{e} \varpi_{2}= \pm 0.005$ in the flow past the conic cylinder with the conic disturbance at $t=380, \lambda=6, W / \lambda=0.1$ and $R e=100$, where the background is the contour of $\omega_{z}$ at $z=0$ with red and blue colors denoting positive and negative values, respectively

$\omega_{x}$ is dominantly negative and positive across the span, while the sign of $\omega_{y}$ is just same as that in the upper shear layer. Moreover, the spanwise vorticity $\omega_{z}$ generated on region RI is convected and transported into the shear layers without any change of sign. This shows that the two sign laws for vorticity are valid in both cases of Figs. 10 and 11. Particularly at $z=0, \lambda$ and $\lambda / 2$ in the upper shear layer, because the sign of induced spanwise vorticity is positive and negative, respectively, according to the theoretical analysis in two induced models, the spanwise vorticity is thus diffused outwards because of its reduced magnitude and concentrated towards the WCP owing to its increased magnitude. Consequently, the upper shear layer is wavy across the span, as shown in Fig. 10c or 11c. Interestingly, this wavy formation is exactly opposite to the geometric disturbance with the minimal diameter at $z=0, \lambda$ and the maximal diameter at $z=\lambda / 2$.

At last we can obtain the main relationships between the three components of vorticity in region R-III only for the case of Fig. 10, except the case of Fig. 11 only with the shear layers at $x>0$ due to the total suppression of spanwise vortices. When the spanwise vorticity is gradually concentrated in the shear layers and finally shed from the shear layers, as well as the streamwise and vertical components of vorticity, the sign of vorticity is invariant in the near wake, as shown in
Fig. 10a-c. Therefore, the first sign variable $\varpi_{1}$ also perfectly agrees with the first sign law in the present region, as shown in Fig. 10d. However as shown in Fig. 10e, the negative sign law appears in the vortex core, still consistent with that in region R-II. Moreover, it is not surprising that the second sign variable $\varpi_{2}$ is positive in the vortex braid due to its stretching mechanism being different from that in the streamwise and vertical vortices, as mentioned above.

In summary, based on above analysis in three flow subregions, two sign laws in the region of $x<0$ appear mainly in region R-I: on and near front cylinder surfaces and near lateral sides of cylinder, even downstream into the separated shear layers to a certain distance under the inertial forces. In regions of R-II and R-III: the separated shear layers and near wake, two sign laws in the region of $x>0$ are still dominant. Particularly, the second sign variable $\varpi_{2}$ is negative mainly in the shear layers and spanwise vortex cores, but positive in the vortex braids in the circumstance of alternately shed spanwise vortices.

\section{Conclusions}

With the purpose of exploring mechanisms in wavy vortices and two sign laws in the shear layers and near wake 

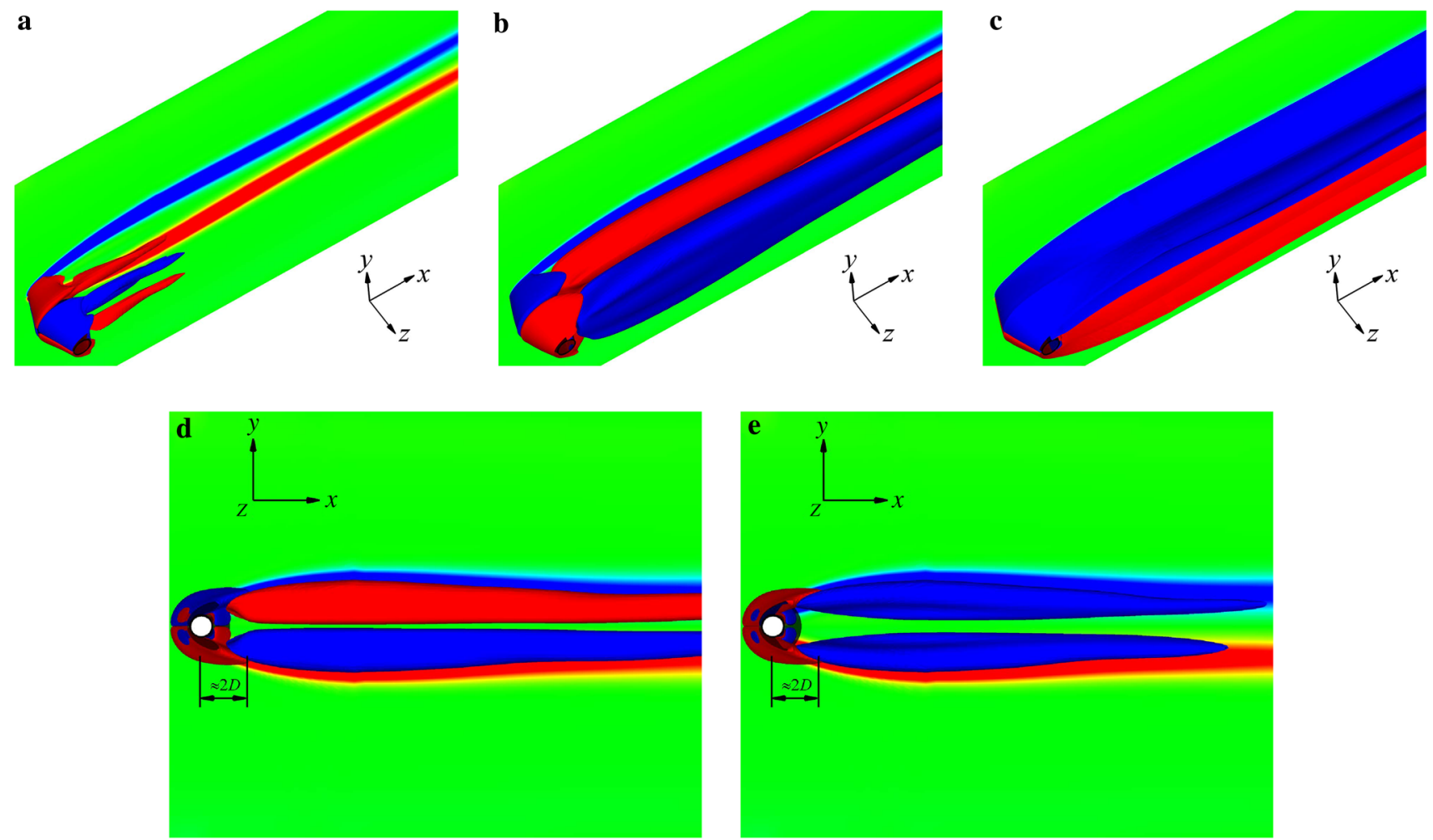

Fig. 11 Iso-surfaces of $\mathbf{a} \omega_{x}= \pm 0.2, \mathbf{b} \omega_{y}= \pm 0.2, \mathbf{c} \omega_{z}= \pm 0.2, \mathbf{d} \varpi_{1}= \pm 0.01$ and $\mathbf{e} \varpi_{2}= \pm 0.01$ in the flow past the circular-section cylinder with the conic disturbance at $t=230, \lambda=4, W / \lambda=0.2$ and $R e=100$, where the background is the contour of $\omega_{z}$ at $z=0$ with red and blue colors denoting positive and negative values, respectively

of the bluff body discovered in previous work [4], the theory of vortex-induced vortex (VIVor) is proposed. The Stokes equations and, subsequently, the simplified vorticity transportation equation are employed, from the viewpoint of investigating vorticity sign, in uniform and incompressible flow past a fixed bluff body. The theory of VIVor is used to illustrate the resultant vorticity field, as well as the velocity field, induced by introducing a specific vortex (or vortex pair) under a particular boundary condition. In the simplest situation, the introduced vortex or vortex pair is uniformly distributed along its own rotating axis, such as $\omega_{x}$ or $\omega_{y}$ introduced along the $x$ - or $y$-axis, respectively. As for the complex spacial distribution of a vortex, the local and bodyfitted coordinate system along its own swirling axis could be established for further analysis. In the present paper, the flow is discussed in the very near wall region, where viscous forces are dominant. Typical bluff bodies are selected in analysis as flat plates and straight bluff bodies with flat stagnation surfaces. Two vortex models, induced by the streamwise and vertical vortex pairs respectively, are proposed on the basis of two kinds of boundary cases, the vorticity generated on walls and over surfaces (disappearing on walls).

According to the basic characteristics of uniform flow past a fixed bluff body, the first sign law for only streamwise and vertical components of vorticity, and the second sign law for three components of vorticity, are obtained. The first sign law reveals the intrinsic physical relationship between streamwise and vertical vorticities, which is independent of the distribution of spanwise vortices in the whole flow field. Moreover, the spanwise vortices, as well as the shear layers and wake width, wavily across the span, are result directly from introduced streamwise or vertical vortices, which induce the local induced spanwise vorticity leading to the original spanwise vorticity increasing or decreasing. Under present circumstances, these two sign laws for vorticity are both independent of the non-dimensional wavelength and Reynolds number. Three flow regions are typically concerned, front cylinder surfaces (R-I), the separated shear layers (R-II) and near wake (R-III). Especially in the shear layers and near wake, the second sign law is generally called the negative sign law due to the specific distributions of spanwise vorticity.

Through the analysis of spacial distributions of vorticity fields in the flow past the conic cylinder at $R e=100$, the VIVor effectively reveals the relationship between different components of vorticity and wavy vortices as a basic physical phenomenon in fluid motion and vortex dynamics. In region R-I, i.e. on and near front surfaces of bluff bodies, usually under 3-D geometric disturbances, in the near lateral sides of cylinder and in the separated shear layers (to a certain 
distance downstream because of inertial forces), the sign of streamwise and vertical components of vorticity, $\omega_{x} \cdot \omega_{y}$, is negative above the wake center plane but positive below the wake center plane, while the sign of three components of vorticity, $\omega_{x} \cdot \omega_{y} \cdot \omega_{z}$, is always positive both above and below the wake center plane. In regions R-II and R-III, the sign of $\omega_{x} \cdot \omega_{y}$ is dominantly positive in the upper shear layer and above the wake center plane but negative in the lower shear layer and below the wake center plane. However, in region R-II, the sign of $\omega_{x} \cdot \omega_{y} \cdot \omega_{z}$ is mainly negative, independent of spanwise vortices alternately shed or totally suppressed, while in region R-III, usually with spanwise vortices alternately shed in the near wake, the sign of $\omega_{x} \cdot \omega_{y} \cdot \omega_{z}$ is always negative in vortex cores but positive in vortex braids due to specific stretching mechanics for the alternatively shedding spanwise vortices and streamwise/vertical vortex pairs, respectively.

Acknowledgements The authors would like to sincerely acknowledge the supports of the National Key Scientific Instrument and Equipment Development Program of China (Grant 2011YQ120048) and the Strategic Priority Research Program of the Chinese Academy of Science (Grant XDB22030101) for the present work.

\section{References}

1. Saffman, P.G., Baker, G.R.: Vortex interactions. Ann. Rev. Fluids Mech. 11, 95-122 (1979)

2. Roshko, A.: On the development of turbulent wakes from vortex streets. NACA Report 1191 (1954)

3. Meiburg, E., Lasheras, J.C.: Experimental and numerical investigation of the three-dimensional transition in plane wakes. J. Fluids Mech. 190, 1-37 (1988)

4. Lin, L.M., Zhong, X.F., Wu, Y.X.: Effect of perforation on flow past a conic cylinder at $R e=100$ : wavy vortex and sign laws. Acta Mech. Sin. (2018). https://doi.org/10.1007/s10409-018-0758-z

5. Wu, J.Z., Ma, H.Y., Zhou, M.D.: Vorticity and Vortex Dynamics. Springer, Berlin (2006)
6. Williamson, C.H.K.: Vortex dynamics in the cylinder wake. Annu. Rev. Fluids Mech. 28, 477-539 (1996)

7. Jiang, H.Y., Cheng, L., Draper, S., et al.: Three-dimensional direct numerical simulation of wake transitions of a circular cylinder. J. Fluids Mech. 801, 353-391 (2016)

8. Jiang, H.Y., Cheng, L., Draper, S., et al.: Prediction of the secondary wake instability of a circular cylinder with direct numerical simulation. Comput. Fluids 149, 172-180 (2017)

9. Jiang, H.Y., Cheng, L., An, H.W.: Three-dimensional wake transition of a square cylinder. J. Fluids Mech. 842, 102-127 (2018)

10. Williamson, C.H.K.: Three-dimensional wake transition. J. Fluids Mech. 328, 345-407 (1996)

11. Luo, S.C., Tong, X.H., Khoo, B.C.: Transition phenomena in the wake of a square cylinder. J. Fluids Struct. 23, 227-248 (2007)

12. Brede, M., Eckelmann, H., Rockwell, D.: On secondary vortices in the cylinder wake. Phys. Fluids 8, 2117-2124 (1996)

13. Darekar, R.M., Sherwin, S.J.: Flow past a square-section cylinder with a wavy stagnation face. J. Fluids Mech. 426, 263-295 (2001)

14. Lin, L.M., Zhong, X.F., Wu, Y.X.: Characteristics for a flow past a circular cylinder with two types of radial disturbances at $R e=100$. Adv. Mater. Res. 871, 107-114 (2014)

15. Yokoi, Y., Kamemoto, K.: Initial stages of a three-dimensional vortex structure existing in a two-dimensional boundary layer separation flow (Visual observation of laminar boundary layer separation over a circular cylinder from the side of a separated region). JSME Int J. Ser. B 36, 201-206 (1993)

16. Kundu, P.K., Cohen, I.M., Dowling, D.R.: Fluid Mechanics, 5th Version. Academic, Berlin (2012) 